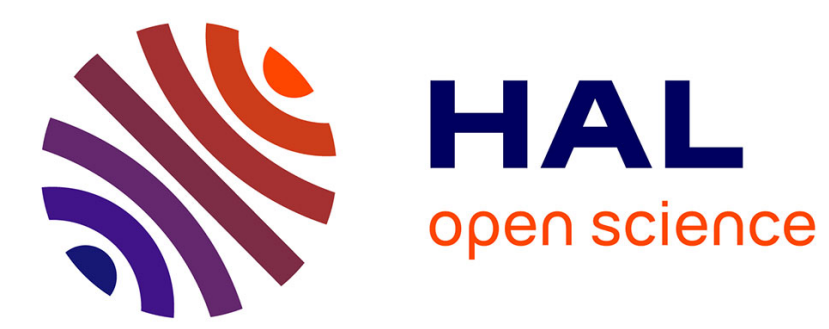

\title{
La citadelle du Caire, un corpus iconographique très particulier
}

\author{
Jean-Luc Arnaud
}

\section{To cite this version:}

Jean-Luc Arnaud. La citadelle du Caire, un corpus iconographique très particulier. Images d'Egypte. De la fresque à la bande dessinée, CEDEJ, pp.291-305, 1991. halshs-00423967

\section{HAL Id: halshs-00423967 https://shs.hal.science/halshs-00423967}

Submitted on 17 Oct 2009

HAL is a multi-disciplinary open access archive for the deposit and dissemination of scientific research documents, whether they are published or not. The documents may come from teaching and research institutions in France or abroad, or from public or private research centers.
L'archive ouverte pluridisciplinaire HAL, est destinée au dépôt et à la diffusion de documents scientifiques de niveau recherche, publiés ou non, émanant des établissements d'enseignement et de recherche français ou étrangers, des laboratoires publics ou privés. 


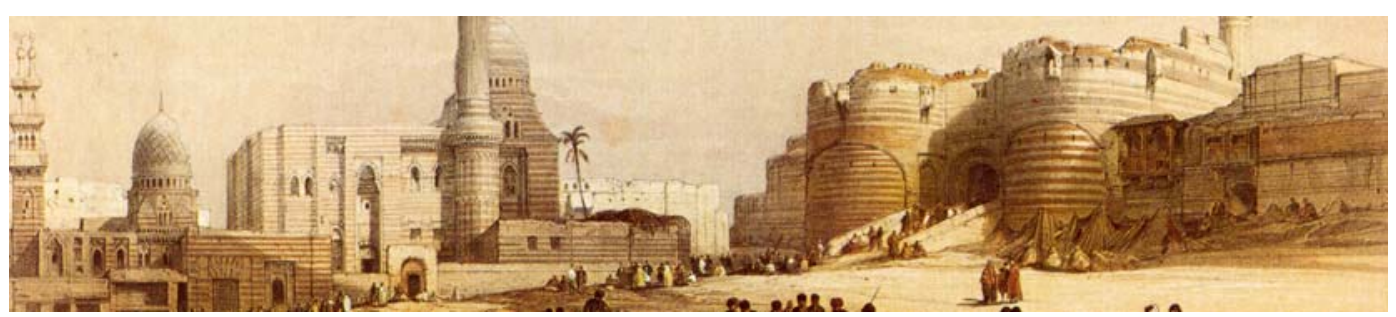

\title{
La citadelle du Caire, un corpus iconographique spécifique
}

\author{
Jean-Luc Arnaud, CNRS, jlarnaud@mmsh.univ-aix.fr \\ D'après « La citadelle du Caire, un corpus iconographique très \\ particulier », in Images d'Egypte. De la fresque à la bande \\ dessinée, Le Caire, CEDEJ, 1991, p. 291-305. \\ Texte remanié, nouvelles figures (Marseille, 2009).
}

\section{Résumé}

Dans l'iconographie moderne et contemporaine de la ville du Caire, la citadelle occupe une place particulière. Autour de deux matrices, dressées depuis des points de vue symétriques, cette iconographie présente deux panoramas - au propre et au figuré - qui résument bien l'histoire de l'Egypte d'une part, et celle du Caire d'autre part.

\section{Abstract}

In the modern and contemporary iconography of the city of Cairo, the citadel occupies a particular place. Around two matrices, drawn up since symmetric points of view, this iconography presents two panoramas which summarize the history of Egypt, on one hand and that of Cairo, on the other hand. 


\section{La citadelle du Caire, un corpus iconographique spécifique}

L'iconographie de la ville du Caire est particulièrement abondante. Depuis le XVIe siècle, la plupart des voyageurs illustrent leurs relations de vues de cette ville. A cette époque, il n'existait pas de plans mais des vues à "vol d'oiseau » qui embrassaient de vastes panoramas. Deux documents de ce type, la vue de Matheo Pagano réalisée au début du XVIe siècle ${ }^{1}$ et celle de Pellegrino Brocardi datée de $1556^{2}$ sont à la base de ces documents. Leurs copies et interprétations constituent la plus grande partie de l'iconographie du Caire jusqu'à une époque récente. A partir du milieu du XVIIIe siècle, les voyageurs sont de plus en plus nombreux et leurs buts se diversifient. En 1761, Carsten Niebuhr publie dans son Voyage en Arabie le premier plan détaillé du Caire ${ }^{3}$. Ce document, malgré son faible niveau de validité (l'auteur s'en explique ${ }^{4}$ ), marque une rupture dans la production iconographique des villes de l'Orient méditerranéen ${ }^{5}$. A la fin du XVIIIe siècle, les grandes vues à «vol d'oiseau » sont abandonnées pour être progressivement remplacées par des plans complétés par des vedute (croquis relevé depuis un point de vue réel qui montre un espace restreint). C'est notamment la sécurité relative des voyageurs à partir du début du XIXe siècle qui permet la réalisation de telles vues $^{6}$. Au même moment, en Europe, l'éclectisme montant dans l'architecture et la décoration dont le style « retour d'Egypte » est un maillon important en France, induit une forte demande de vues des villes orientales. Les gravures d'Istanbul dressées par Bartlett en $1839^{7}$, celles de David Roberts pour l'Egypte publiées la même année ${ }^{8}$ et leurs multiples rééditions dans des formats divers ou encore, le succès de

\footnotetext{
${ }^{1}$ J.-C. Garcin, «Une carte du Caire vers la fin du sultanat de Qaytbay », Annales islamologiques, 17, Le Caire, 1981, p. 272-285.

2 L. Micara, « Il Cairo nella "Chorographia" di Pellegrino Brocardi (1556) », Storia della città, 46. Il mondo islamico Immagini e ricerche, 1989, p. 7-18.

${ }^{3}$ Le plan du Caire publié par Pococke en 1843 représente seulement le périmètre de la ville, celui de Niebuhr (1761) indique les principales rues et l'occupation des zones non urbanisées. R. Pococke, Description of the East and some other Countries, Londres, 1743-1748 ; C. Niebuhr, Voyage en Arabie et en d'autres païs circonvoisins (1761), Amsterdam, 1776, 2 vol.

${ }^{4} \mathrm{C}$. Niebuhr explique sa méthode de relevé « au pas et à la boussole » et indique le niveau de validité de son plan de Constantinople. Ce plan est le premier présenté dans l'ouvrage, la méthode citée semble s'appliquer aussi aux plans qui suivent. Ibid., p. 19.

${ }^{5}$ C. Niebuhr publie trois représentations du Caire : deux plans et une veduta. Ibid.

${ }^{6}$ Ibid., p. 19. Melling, Barbié du Bocage, Voyage pittoresque de Constantinople et des rives du Bosphore, Paris, 1819. Au sujet de la sécurité récente des voyageurs et des dessinateurs voir l'explication de la pl. 22.

${ }^{7}$ M. Pardoe, Les beautés du Bosphore orné d'une suite de vues du Bosphore et de ses environs, Londres, 1839.

${ }^{8}$ D. Roberts, The Holy Land, Syria, Egypt and Nubia, Londres, 1839.
} 
Fig. 1. La mosquée Sultan Hassan et la place Rumaliyya à la fin du XVIIIe siècle*

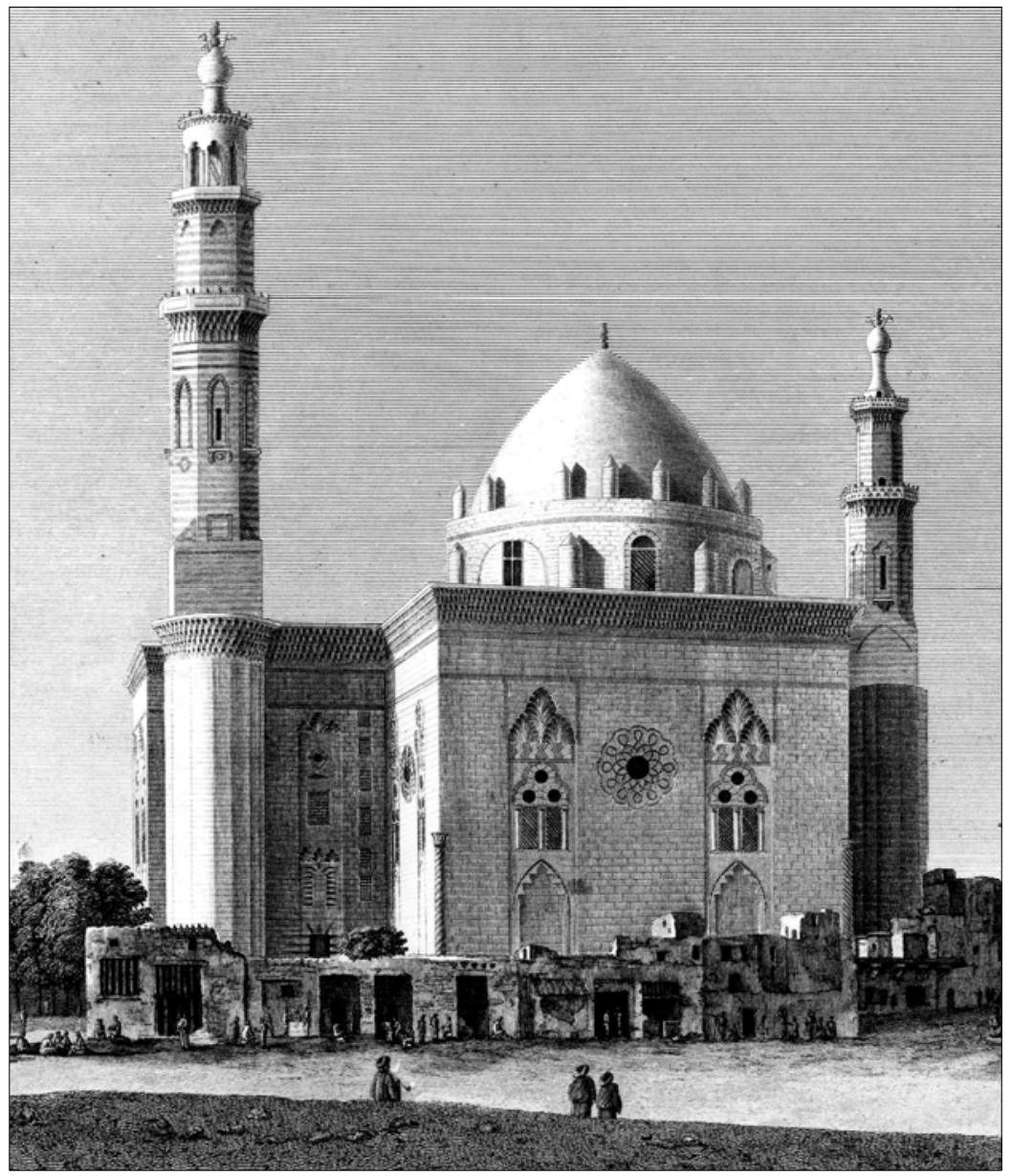

* Description de l'Egypte - Etat moderne, Paris, 1909, extrait de la pl. 32.

l'ouvrage de Vivant Denon ${ }^{9}$ constituent autant d'exemples de cet engouement de l'Europe pour les vues des villes orientales.

Face à l'importance de cette documentation, l'historien de l'urbanisation a besoin de repères. Un premier classement du corpus pour une utilisation comme source pour l'histoire des espaces urbains conduit à plusieurs remarques. Il est notable que les copies sont particulièrement nombreuses. Les plus récurrentes sont celles des vues à «vol d'oiseau » des XVIe, XVIIe et du début du XVIIIe siècle. Pour cette période, on compte peu de matrices. Les copies sont identifiées sur la base de comparaisons systématiques des dispositions, du point et de l'angle de vue de chaque document. Les vedute du XIXe siècle posent d'autres problèmes. Le corpus est beaucoup plus large et plus varié et, durant cette période, les copistes ne se sont pas privés de réinterprétations, depuis la transformation des premiers plans des vues ou de leurs fonds jusqu'au retournement pur et simple des documents. Ces opérations, facilitées par la technique de la gravure, ont permis la démultiplication des documents à partir d'un même original. On trouve ainsi deux vues de la ville de Mytilène légendées « Port Nord » et « Port Sud » qui représentent

\footnotetext{
9 J.-E. Goby, «Les quarante éditions, traductions et adaptations du "Voyage dans la Basse et la Haute Egypte" de Vivant Denon », Cahiers d'histoire égyptienne, série IV, fasc. 5-6, déc. 1952, p. 1-29.
} 
Fig. 2. La mosquée Sultan Hassan et la place Rumaliyya vers 1830*

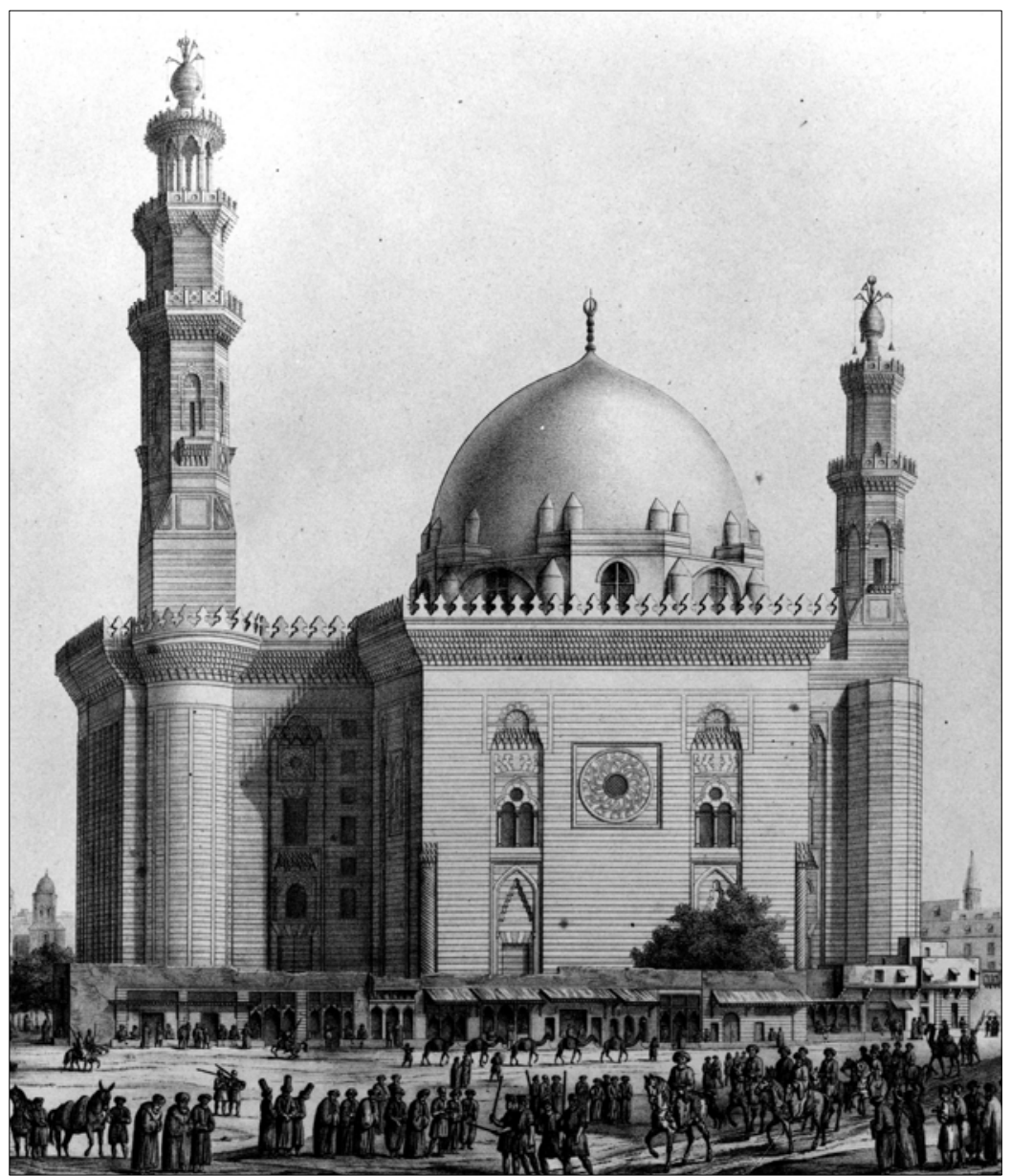

* P. X. Coste, Architecture arabe des monuments du Kaire, Paris, 1839, extrait de la pl. 26.

le même espace gravé à l'endroit et à l'envers. La supercherie est complétée par la différence du graphisme et la symétrie relative des deux ports ${ }^{10}$.

Ces vedute, petits croquis qui semblent être pris «sur le vif ", réservent des surprises lors d'une investigation plus poussée. On pourrait imaginer, selon les règles de la perspective conique établies au XVe siècle, des représentations relevées depuis un point de vue unique. Les tentatives de restitution montrent, au contraire, que les documents publiés résultent du montage de plusieurs croquis et que l'auteur s'est déplacé entre chaque élément relevé de telle manière que les règles de la perspective sont peu respectées. Ainsi, le plus souvent, il s'avère impossible de procéder à la restitution de ces documents. Les vues des pyramides de Giza publiées dans la Description de l'Egypte aux planches 22 et $17-3^{11}$ témoignent de l'importante liberté des dessinateurs vis-à-vis de l'objet de leur travail et des difficultés que ce type de document présente pour être utilisé comme source pour l'histoire de l'espace. Ces deux vues, relevées depuis l'île de Roda, montrent des pyramides construites au niveau des terres agricoles (alors que le plateau de

\footnotetext{
${ }^{10}$ D. Garudis, Mytilène et son évolution urbaine, mémoire de fin d'études d'architecture, Nanterre, 1985. p. 25 sq.

${ }^{11}$ Description de l'Egypte - Etat moderne, Paris, 1809.
} 
Fig. 3.Panorama du Caire depuis la citadelle, au fond à gauche : les pyramides de Giza*

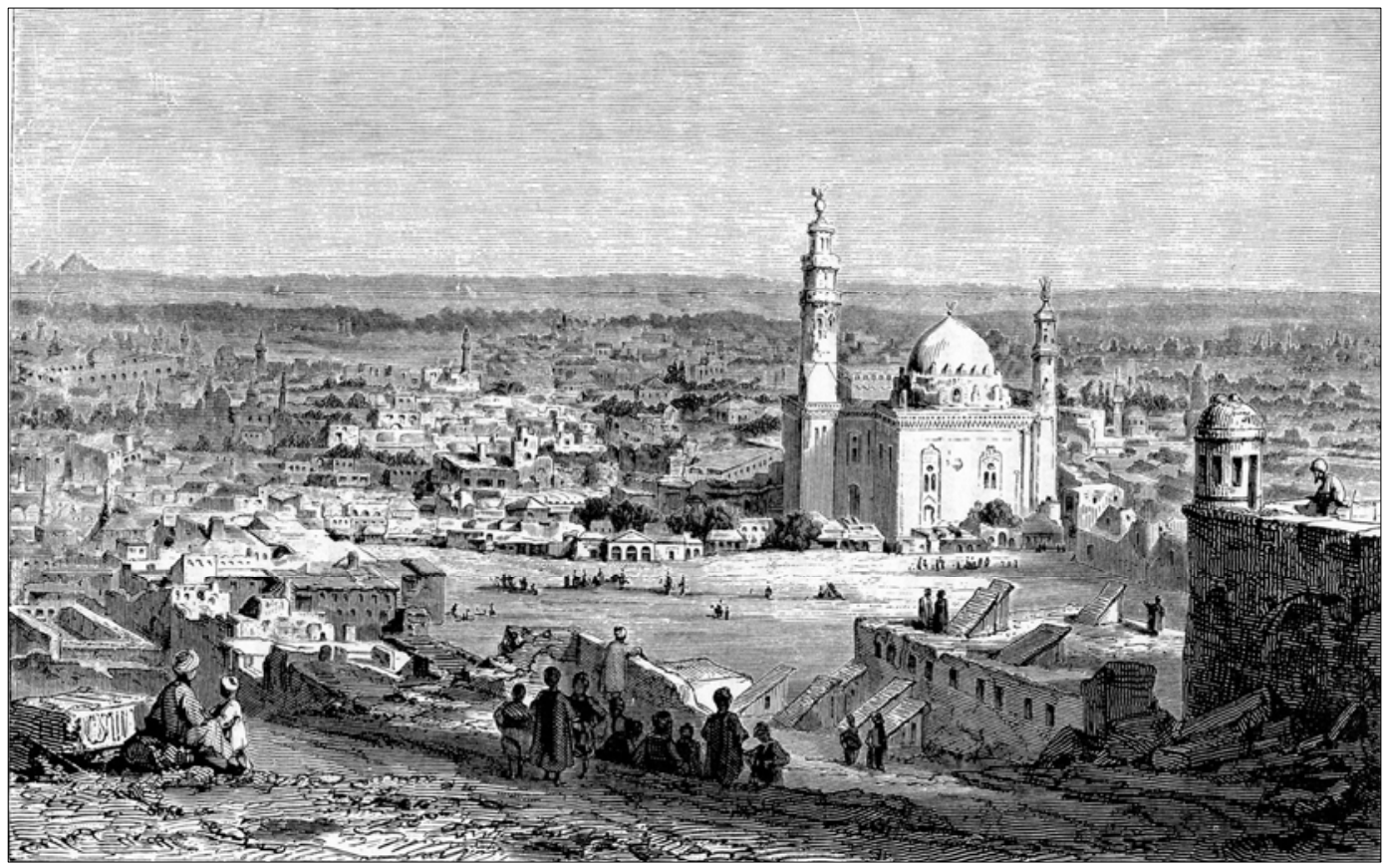

* E. W. LANE, An account of the Manners and Customs of the Modern Egyptians, Londres, 1837.

Giza est situé à plus de 40 mètres au-dessus de ces terres). Elles présentent aussi une importante différence d'échelle ; enfin, les positions relatives des pyramides ne sont pas identiques selon les deux documents. On constate des déformations du même ordre dans les vedute de Constantinople du milieu du XIXe siècle ${ }^{12}$.

La diffusion de la photographie à partir de 1850 et, pour l'Egypte, la publication de l'atlas photographique de Teynard en $1858^{13}$ évacue doublement le problème de la validation des documents. Tout d'abord, les vedute sont progressivement remplacées par des photographies ; ensuite, les dessinateurs travaillent d'après des originaux photographiques et de plus en plus rarement sur le terrain. Bien que ces documents soient souvent retouchés, les auteurs en respectent les dispositions générales.

Dans un second temps, l'historien des espaces urbains cherche à identifier, localiser et dater les matrices. Les nombreuses copies ou interprétations qui ont décuplé l'iconographie du XIXe siècle ne facilitent pas le travail. De plus, il est souvent impossible d'identifier l'auteur et donc la date de relevé des originaux. Seules les vues issues de recueils dont l'auteur, dessinateur ou photographe, est bien repéré, sont facilement datables. La localisation des vues est d'autant plus complexe que l'espace représenté est apparemment banal, le caractère laconique des légendes « Rue du quartier arabe » ou « Ville indigène » permet rarement l'identification des points de vue.

Enfin, l'utilisation de ces documents pour l'histoire des espaces urbains nécessite des comparaisons diachroniques qui supposent des points comparables de prise de vue. Il

\footnotetext{
12 J.-L. Arnaud, Sources iconographiques d'histoire de l'espace urbain, Mém. de DESS, Paris, 1988, vol. I, p. 25-26.

${ }^{13}$ F. Teynard, Egypte et Nubie, sites et monuments les plus intéressants pour l'étude de l'art et de l'histoire, Paris, 1858.
} 
Fig. 4. La place de la citadelle - Qaramaydan - en 1798

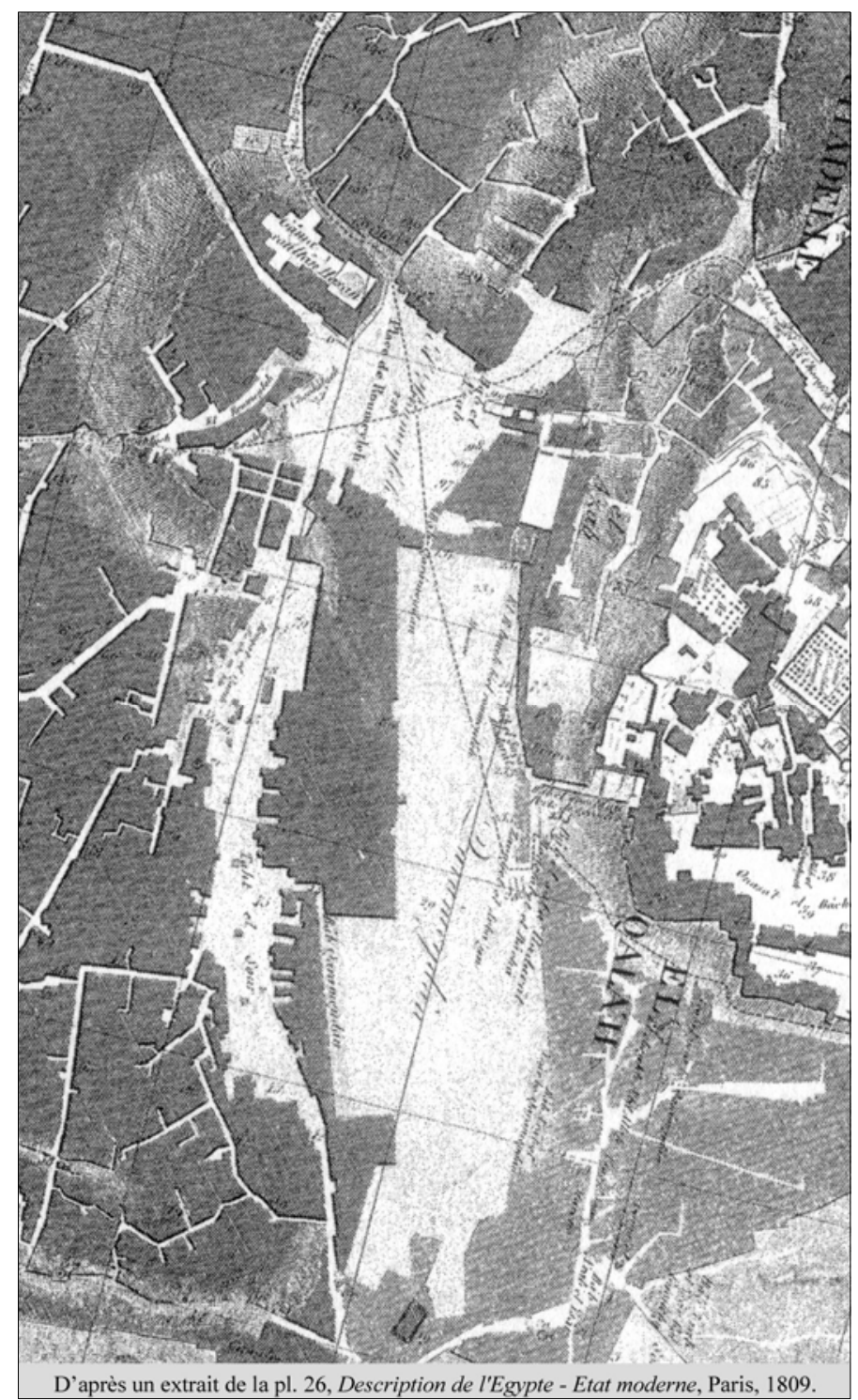

pourrait sembler difficile de trouver dans une ville aussi diverse et variée que Le Caire, sur une période de plusieurs décennies, des documents dressés depuis le même point de vue. L'existence de belvédères ne constitue pas une condition suffisante à la réalisation de cette opportunité. Elle nécessite des dispositions particulières de l'espace dans la mesure où les règles de composition des images exigent une structuration en plans successifs et notamment un premier plan remarquable. Cette nécessité explique pourquoi les vues prises depuis les minarets (malgré leur nombre) sont assez rares. Ils ne présentent généralement qu'une série assez uniforme de terrasses d'où émergent de temps à autre des minarets qui ne forment pas une masse suffisante pour équilibrer la composition des vues.

Au Caire, la citadelle semble avoir été le point de vue privilégié pour de multiples documents iconographiques réalisés et durant une longue période ${ }^{14}$. Entourée d'un côté

\footnotetext{
${ }^{14}$ Les vues de la citadelle représentent exactement $10 \%$ du fonds iconographique de vues du Caire conservé à l'Observatoire urbain du Caire contemporain (CEDEJ).
} 
Fig. 5. La place de la citadelle en 1874

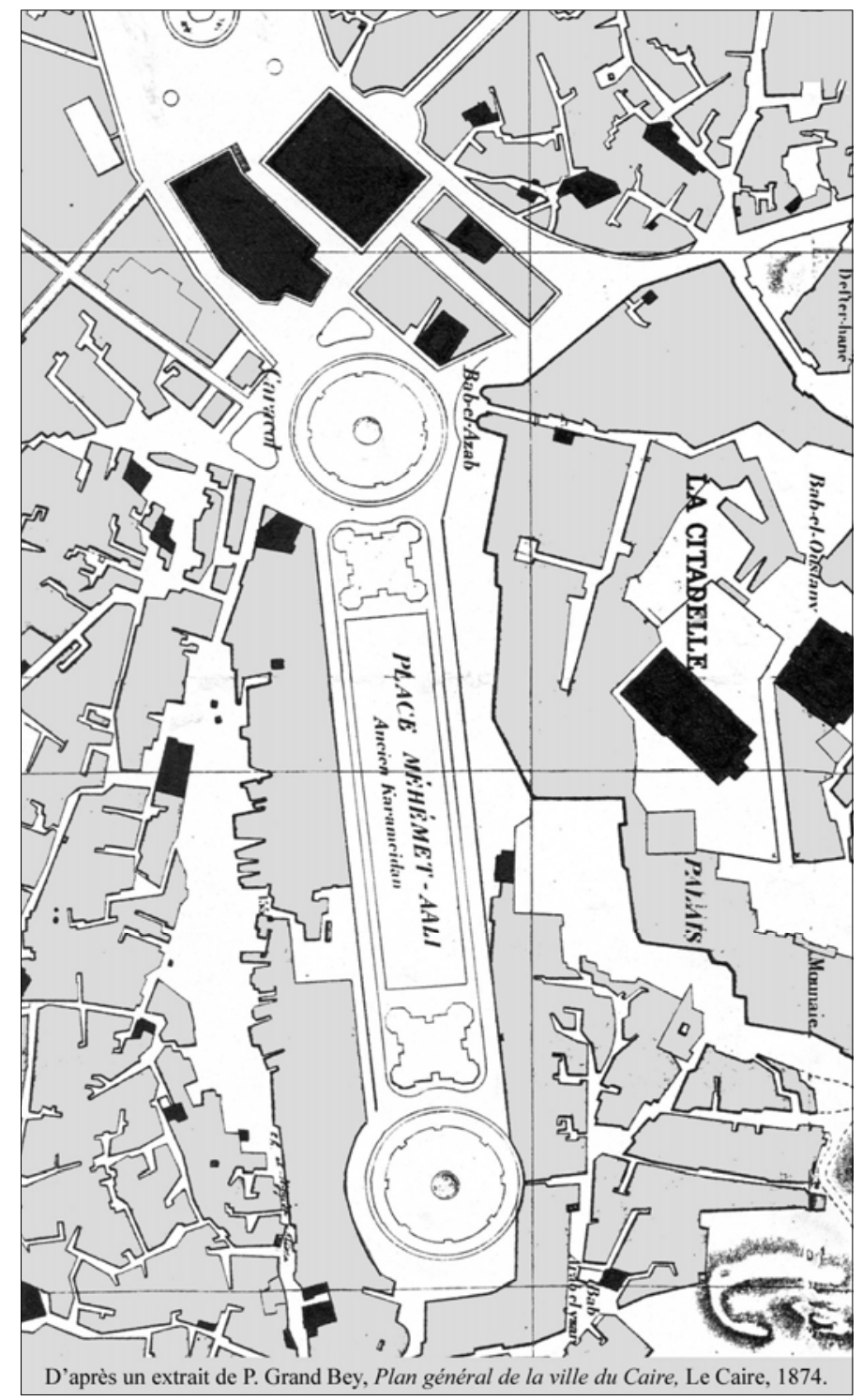

par les hauteurs du Muqattam, au sud par le cimetière Imam al-Chafa'i et à l'ouest par une longue place, la citadelle est un des rares points hauts de la ville. La proximité de la monumentale mosquée Sultan Hassan située, au nord de la place, et le vis-à-vis qu'elle entretient avec les fortifications de la citadelle ont aussi contribué au redoublement de l'intérêt iconographique porté à ce quartier. Avant d'établir une typologie de ces documents, il faut en distinguer deux classes : ceux qui sont vus depuis la citadelle et ceux qui la représentent. Ces deux catégories correspondent à une double particularité de la citadelle. D'une part, grâce à sa hauteur et au dégagement de sa place, la citadelle permet de prendre des vues sur un très large panorama ; d'autre part, elle se donne à voir depuis de nombreux points de la ville (sa terrasse culmine à plus de 60 mètres au-dessus de la ville). Malgré les récents développements de l'urbanisation, la mosquée Muhammad 'Ali, qui surmonte cette terrasse, reste un point de repère important. 


\section{Depuis la citadelle - 1}

L'attrait principal de la citadelle est l'immense panorama que l'on y découvre. Des deux côtés d'un petit pavillon vert, séparé du reste des bâtiments et qu'affectionnait, dit-on, Mohamed-Ali, on jouit d'une admirable vue. Devant soi, immédiatement à ses pieds, est la place Roumeïlèh, avec la mosquée du sultan Hassan; derrière celle-ci, l'ancien harem d'Abbas-Pacha, un peu plus loin à gauche est la mosquée de Touloun, reconnaissable à son gros minaret; plus au S., la grande place Karameïdan, bordée de casernes; au delà de ce premier plan, l'immense ville se déploie toute hérissée de minarets. Au milieu de la masse confuse de ses terrasses, les rues n'apparaissent que comme de sombres fissures. De grands palais blancs se montrent à côté des arbres touffus de l'Esbékyèh, qui semble toucher à Boulak. Entre les palmiers des plantations d'Ibrahim-Pacha, on aperçoit le palais de ce prince et Kassr el-Aïny. Au delà le Nil coule lentement dans son large lit, bordé d'une ligne de riche verdure, et va se perdre dans les plaines du Delta; puis au dernier plan, tout au fond de l'horizon, la vue rencontre la masse encore imposante, même à cette distance de 4 lieues, des pyramides de Gizèh, d'Aboussir et de Sakkarah, qui se détachent sur le fond rougeâtre du désert. En reportant le regard du côté du Mokattam, sur un plan plus rapproché, on verra le vieux Caire, le grand aqueduc, qui vient à la citadelle à travers l'immense champ de décombres de l'ancienne Fostat; la nécropole de I'Imam Chafy, avec toutes ses coupoles et tous ses minarets; les pentes $d u$ Mokattam, avec une autre nécropole; enfin le fortin qui couvre le sommet du Gébel-Giouchi (autre nom du Mokattam), et qui commande la citadelle elle même. C'est le matin qu'il faut venir admirer ce panorama; le soir, les brumes couvrent la plaine des pyramides.

Extrait de A. Joanne et E. Isambert, Itinéraire de l'Orient, Paris, 1861. p. 980. Cette description est mentionnée dans le cadre de la description de la citadelle, entre la présentation de la mosquée de Mohamed Ali et celle du puits de Joseph.

Le grand nombre de vues de la citadelle ne dépend pas seulement de la topographie, la valeur symbolique des constructions qui y sont assemblées et leur mise en perspective historique contribue aussi à l'engouement des dessinateurs puis des photographes. La citadelle n'est pas seulement un point élevé, construite par Saladin au XIIe siècle, elle fut le siège principal du pouvoir jusqu'au milieu du XIXe siècle pour devenir un centre d'ateliers, d'écoles et de ministères tout en restant totalement contrôlée par l'autorité publique. Le grand nombre de palais, de mosquées et d'autres constructions qui s'y trouvent témoignent de cette longue succession. Depuis quelques années seulement la citadelle n'est plus placée sous le contrôle du ministère de la Défense et, actuellement, le musée de l'Armée occupe une partie non négligeable de ses constructions. Lieu du pouvoir et de l'expression de sa puissance, la citadelle renvoie directement à un autre lieu d'expression du pouvoir, beaucoup plus ancien mais constituant principal de l'image de l'Egypte et du Caire dans l'imaginaire collectif : les pyramides de Giza dont le contour émerge du panorama depuis la terrasse de la citadelle. Les silhouettes fugitives qui 
Fig. 6. La place Qaramaydan et Bab al-‘Azab à la fin des années 1830*

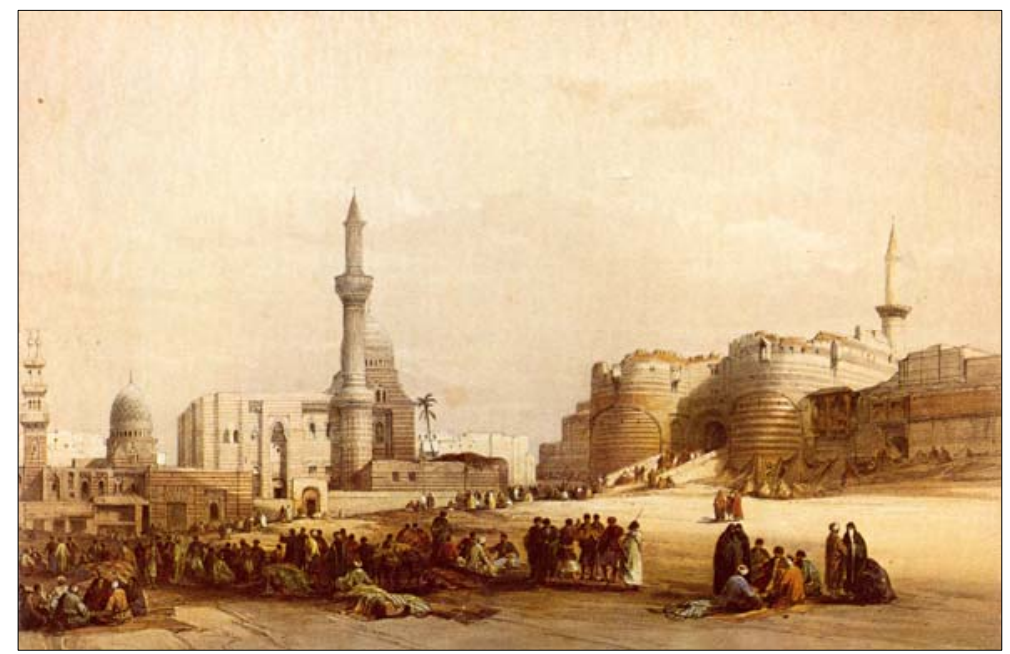

* D. Roberts, Londres, 1839.

Fig. 7. Les mosquées Sultan Hassan et Al-Rifa'i (en construction) vers 1907*

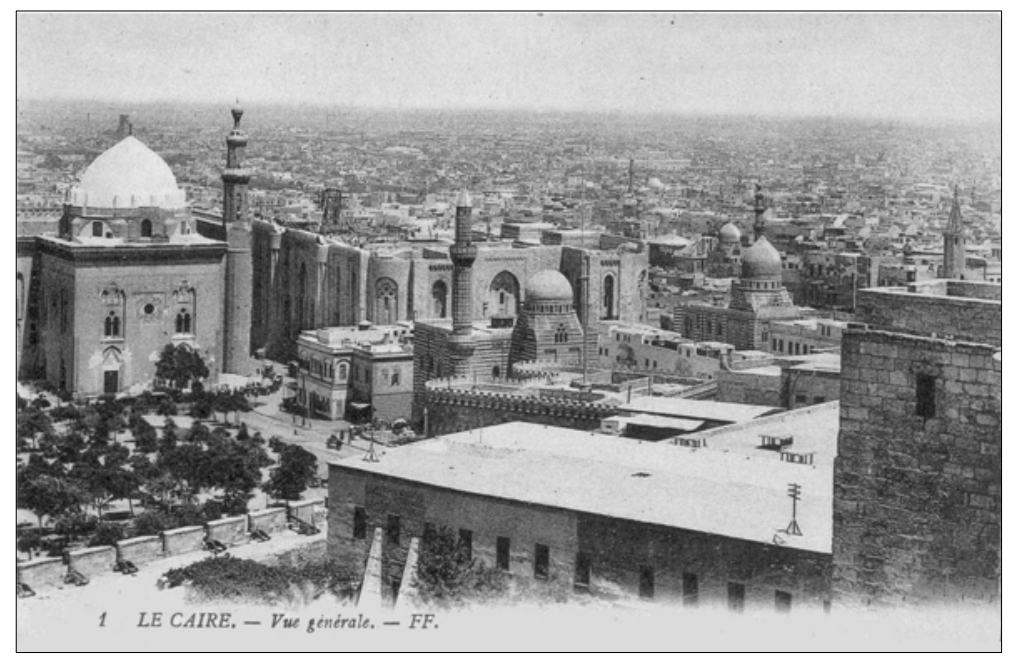

* Coll. JLA.

marquent aujourd'hui l'horizon des visiteurs n'en sont pas moins symboliques; les relations de voyage des siècles précédents ne manquent jamais de mentionner les pyramides dans leurs descriptions de la citadelle. De la même manière, les guides destinés aux voyageurs, depuis celui de Joanne et Isambert (1861) jusqu'aux plus récents, poursuivent cette tradition en donnant systématiquement une description du panorama vu depuis la citadelle ${ }^{15}$. Ainsi, depuis la cour de la mosquée Muhammad 'Ali jusqu'au plateau de Giza, ce sont 4000 ans d'histoire de l'Egypte qui s'étalent aux pieds des voyageurs. Quatre millénaires jalonnés par la masse imposante de la mosquée Sultan Hassan construite au XIVe siècle, par l'enceinte de la mosquée Ibn Tulun (élevée au IXe

\footnotetext{
${ }^{15}$ En 1806, F.R. de Châteaubriant, Itinéraire de Paris à Jérusalem, Paris, 1863, vol. II, p. 134. En 1843, G. de Nerval, Voyage en Orient, Paris, 1927, vol. II, p. 344. En 1861, A. Joanne et E. Isambert, Itinéraire de l'Orient, Paris, 1861, p. 980. En 1868, F. Levernay, Guide général de l'Egypte, Alexandrie, 1868, p. 148149. En 1908, Le Caire et ses environs, Paris, 1909, p. 46. Voir aussi les deux encadrés hors texte.
} 
Fig. 8. La citadelle vue depuis le cimetière Imam al-Chafa'i vers 1900*

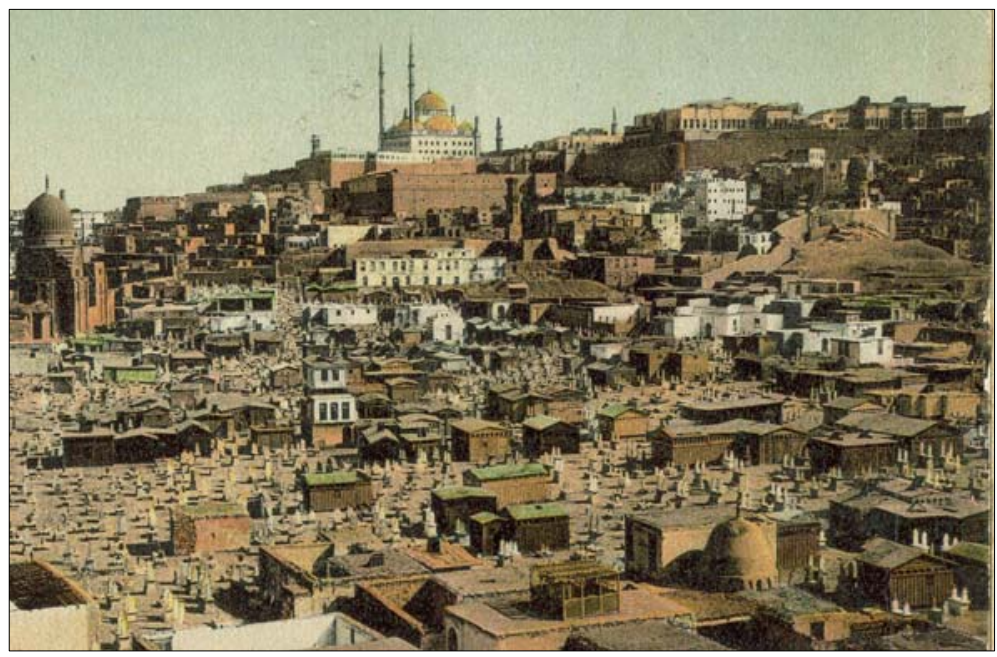

Fig. 9. Bab al-'Azab Entrée inférieure de la citadelle, vers 1980*

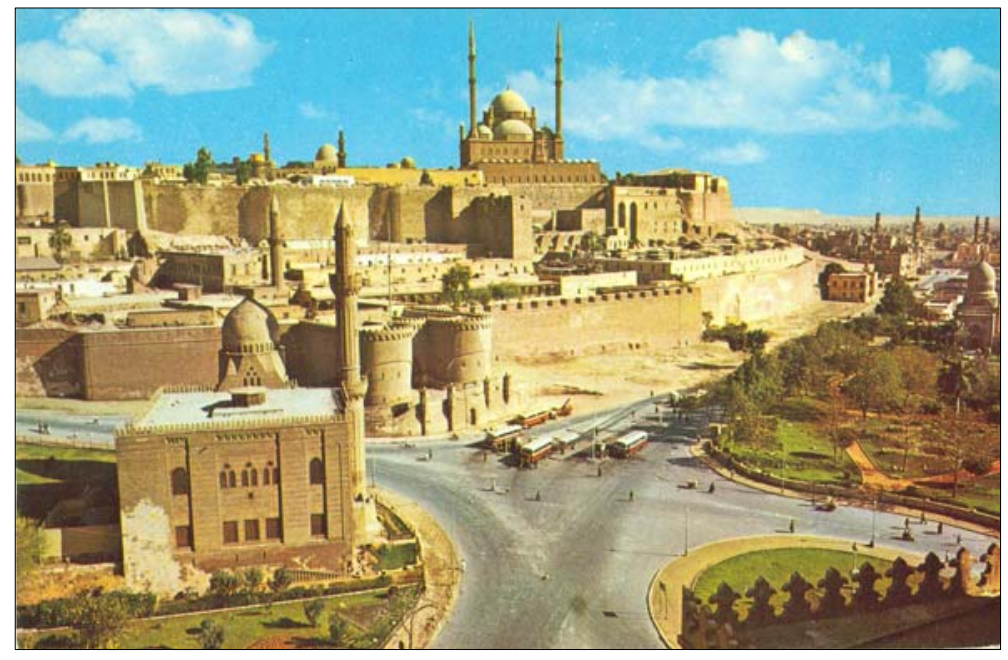

* Coll. JLA.

siècle) et par la multitude des minarets et des coupoles qui émergent des terrasses d'une ville dont les limites disparaissent à l'horizon. Quatre millénaires complétés par la mosquée Al-Rifa'i (achevée en 1911), les aménagements récents de la place située au pied de la citadelle, la voie rapide Salah-Salim ouverte à travers les cimetières vers 1960 et enfin, les silhouettes des tours en construction sur la corniche de Maadi. L'histoire complète de l'Egypte est résumée dans ce panorama. Faut-il s'étonner que la citadelle constitue l'étape cairote principale des voyageurs pressés de la fin du $\mathrm{XX}^{\mathrm{e}}$ siècle ?

Cette image de la ville, complète et partielle à la fois, est à rapprocher des vues à « vol d'oiseau » qui ont illustré les relations de voyage jusqu'à la fin du XVIIIe siècle.

Ces vues montraient, émergeant d'un tissu urbain représenté uniformément, les principaux bâtiments remarquables de la ville dont la liste correspond à peu de chose près à ceux qui sont identifiables aujourd'hui depuis la citadelle. En outre, la technique de construction de la vue à « vol d'oiseau » - prise depuis un point de vue fictif -, autorise des changements d'échelle et d'angle qui permettent de mettre en valeur les lieux importants (les pyramides 


\section{Depuis la citadelle - 2}

De la terrasse [de la citadelle] se déroule un panorama magnifique. Le Caire sous vos pieds avec ses 400 mosquées, ses jardins qui découpent le ton monotone et sombre des maisons, les vastes plantations et les belles avenues qui couvrent l'espace compris entre la ville et le Nil, Boulac, le Vieux Caire, Rhoda, les plis jaunâtres du grand fleuve; plus loin, en face, Gizeh et ses pyramides, ces colosses que ni le fleuve, ni les hommes, ni le temps n'ont pu détruire et qui vous invitent à venir admirer le lever du soleil du haut de leur aiguille; plus loin encore vers le Sud les quinze pyramides de Sakarah, les monts Lybiens et les plaines immenses $d u$ désert. Là votre imagination se complet à exhumer des fastes du vieux monde les souvenirs si attachants d'une histoire qui, d'abord légendaire, se dégage peu à peu des langes du vieil âge, devient certaine, brillante et montre à l'admiration les peuples et les rois qui ont laissé tant d'impérissables monuments. Voyez-vous les Pharaons remplissant la vallée du Nil d'immenses cités, peuplées d'oeuvres d'art qui étonnent encore même par leurs débris; les Perses bâtissant, vers l'emplacement du vieux Caire, une nouvelle Babylone qui a péri comme la première; Joseph creusant les canaux et fécondant l'Egypte; les juifs captifs construisant les pyramides de Daschour ; la fille de Pharaon se baignant dans le Nil en face de Rhoda et recueillant de ses mains le jeune Moïse voguant à la dérive dans son frêle berceau; la sainte famille fuyant la Judée et venant chercher un refuge à quelques pas de vous, au bas de la pente abrupte qui descend au vieux Caire ; divers peuples envahissant le pays, les uns édifiant, les autres détruisant ; le christianisme régnant à son tour et peuplant la Thébaïde de solitaires ; puis le Croissant remplaçant la Croix et dominant jusqu'au moment où le grand MéhémetAli, comprenant son pays et son temps, a recommencé une ère de prospérité et de gloire qui continue de briller aujourd'hui sur l'Egypte.

Extrait de F. Levernay, Guide général de l'Egypte, Alexandrie, 1868, p. 148-149.

de Giza qui sont absentes de la vue définitive de P. Brocardi sont mentionnées sur son croquis de manière très disproportionné $\mathrm{e}^{16}$ ). La veduta, même si elle autorise certains « écarts » par rapport aux règles de la perspective conique, se présente, par définition, comme une vue dressée depuis un point de vue réel, elle ne peut donc pas montrer la totalité du panorama qui s'offre depuis la citadelle. De même la photographie, soumise aux conditions atmosphériques et à l'angle d'ouverture des objectifs, permet seulement des prises de vue partielles.

Cette image transhistorique n'est donc pratiquement pas reproductible dans son intégralité. Ce sont seulement des éléments ponctuels de ce panorama qui sont donnés par l'iconographie contemporaine. La proximité de la mosquée Sultan Hassan et sa monumentalité n'ont pas échappées aux observateurs et c'est de ce côté-là que les dessinateurs et les photographes des XIXe et XXe siècles se sont le plus souvent tournés ${ }^{17}$.

\footnotetext{
${ }^{16}$ L. Micara, op. cit., p. 9.

${ }^{17}$ Dans la vue générale du Caire prise depuis la citadelle et publiée par E. W. Lane dans les premières pages de son livre, la mosquée Sultan Hassan et la place Rumaliyya qui la précède constituent bien l'objet principal
} 
Fig. 10. Les mosquées Sultan Hassan et Al-Rifa'i vers 1970*

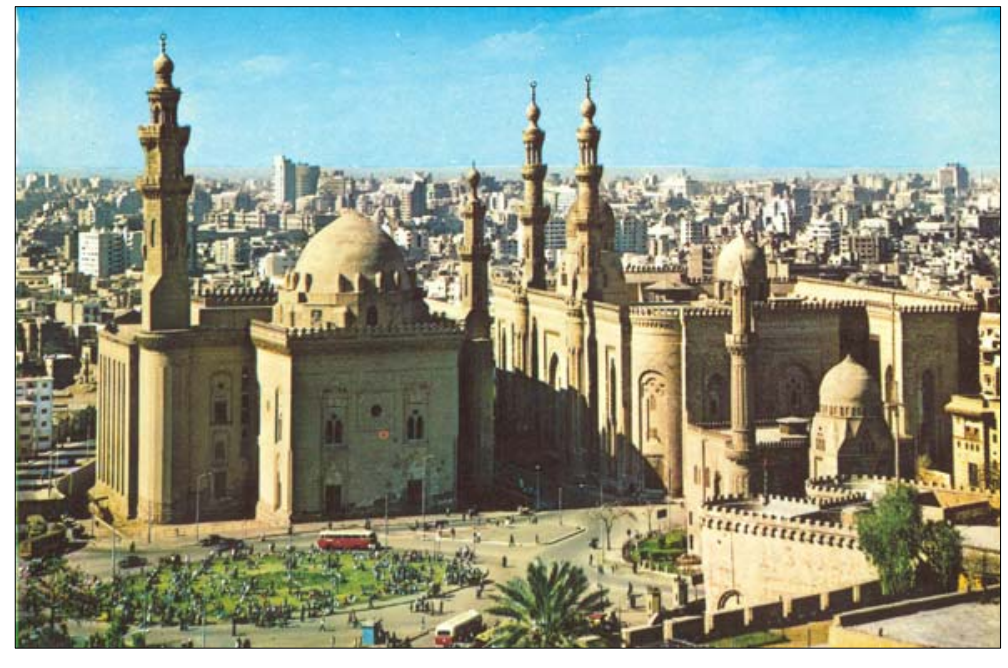

* Coll. JLA.

La terrasse de la citadelle offre vers la qibla de cette mosquée une vue qui semble avoir comblé les voyageurs. Une série ininterrompue de documents représente cette mosquée, depuis le début du XIXe siècle jusqu'à aujourd'hui. Les cartes postales ont remplacé les gravures mais le point de vue est resté le même.

Cette série est d'autant plus intéressante que le quartier a subi de nombreuses transformations. Le remaniement des places situées au pied de la citadelle a été engagé dans la première phase des grands travaux de transformation du Caire entrepris sous Ismaïl en 1867. Dès la fin de l'année 1869, les deux places Rumaliyya et Qaramaydan sont réunies, les boutiques construites au pied de la mosquée Sultan Hassan sont détruites et l'entrée basse de la citadelle, Bab al-'Azab, est en cours de restauration ${ }^{18}$.

La construction de la mosquée Al-Rifa'i, engagée en 1869, mais complétée après plusieurs péripéties en 1911 seulement, vient à nouveau transformer le site au début du siècle ${ }^{19}$. Enfin, vers 1915, la démolition d’un grand bâtiment situé à côté de la mosquée Mahmudiyya, au débouché de la rue Muhammad 'Ali, a complété les grandes opérations de transformation de la place. Ces modifications successives sont bien documentées par l'iconographie, mais elles sont aussi mentionnées, de manière souvent plus complète, dans les plans ou les sources écrites. A une autre échelle, les aménagements des espaces publics sont rarement mentionnés de manière détaillée dans les sources écrites alors que l'iconographie en donne des images très précises. Ainsi les gravures puis les photographies permettent de suivre précisément les états successifs du rond point Nord de

de la vue, mais les pyramides de Giza sont indiquées à l'horizon à gauche, au-dessus de l'enceinte de la mosquée Ibn Tulun. Or, il est impossible d'aligner ces constructions depuis la citadelle. Ce collage des pyramides vient en quelque sorte confirmer que la ville représentée est bien celle du Caire. E. W. Lane, Manners and Customs of the Modern Egyptians, La Hague, Londres, Le Caire, 1981, p. 13.

${ }^{18}$ E. Fromentin, Voyage en Egypte, Paris, 1935, p. 158. Il voyage en 1869.

${ }^{19}$ M. Hertz Bey, La mosquée El-Rifaï au Caire, s.l., 1911, p. 13 sq. 
la place de la citadelle dont le terrain en pente a contribué, par essais et erreurs, à la multiplication des tentatives d'aménagement..

Mais la citadelle n'est pas seulement un point de prise de vue, elle est aussi l'objet d'une iconographie abondante grâce au dégagement de ses environs proches et au vis-àvis qu'elle entretient avec les deux grandes mosquées du nord de la place. La variété des angles sous lesquels elle apparaît a largement contribué au développement de son iconographie.

Les divers documents représentant la citadelle ont un point commun qui mérite d'être remarqué. Que les vues soient prises depuis les hauteurs du Muqattam, depuis le cimetière Imam al-Chafa'i ou depuis la mosquée Ibn Tulun, leur composition est toujours organisée autour de la mosquée Muhammad 'Ali dont la silhouette, qui occupe la partie centrale, se découpe sur le ciel ou sur la masse confuse du tissu urbain banal. La présence de cette mosquée dans les vues de la citadelle est devenue quasiment obligatoire. Sa construction (en 1843) a entraîné le déplacement du point habituel de vue de la porte Bab al-'Azab. Avant cette date, le point de vue le plus fréquemment utilisé pour représenter cette porte était l'angle occidental de la place Rumaliyya, la porte et la mosquée Mahmudiyya en constituaient alors les éléments principaux. Depuis le milieu du XIXe siècle, les auteurs des représentations de la même porte se sont déplacés au pied de la mosquée Sultan Hassan de manière à montrer, derrière le premier plan constitué par la porte, la mosquée Muhammad 'Ali récemment construite.

Les vues de la citadelle les plus récurrentes sont prises depuis la terrasse de la mosquée Sultan Hassan ou celle de la mosquée Al-Rifa'i. Ces points de vue permettent de saisir, couronné par l'incontournable mosquée Muhammad 'Ali, un vaste panorama sur les terrasses successives de la forteresse. Le premier plan est occupé à gauche par la mosquée Mahmudiyya, à droite la vue s'ouvre vers les plantations du centre de la place. Symétrique de celle qui, depuis la citadelle, regarde les mosquées Sultan Hassan et Rifa'i, cette vue présente plusieurs avantages (fig. 8). Centrée sur la mosquée Muhammad 'Ali, elle montre aussi la coupole de la mosquée Muhammad al-Nasir Ibn Qalawun, deux minarets, des bâtiments d'architecture domestique, plusieurs terrasses, la porte Al-'Azab et les fortifications. Cette vue semble condenser l'ensemble de la citadelle. La place, le rondpoint et le jardin indiquent l'insertion de l'ensemble monumental dans le tissu urbain récent tandis que la mosquée Mahmudiyya au premier plan et les minarets du cimetière Imam al-Chafa'i au fond témoignent de l'étendue relative d'une ville riche en monuments.

A l'instar de la vue symétrique qui réunit l'ensemble des attributs de l'Egypte, cette vue peut être considérée comme un concentré de l'histoire du Caire. Depuis les conquêtes de Saladin jusqu'aux travaux récents de voirie et de restauration, en passant par le point culminant de la composition des réformes de Muhammad 'Ali. Sa mosquée est d'autant plus remarquable qu'elle est la seule de ce type au Caire et qu'elle est présentée comme une copie de la célèbre Mosquée Bleue d'Istanbul.

Images de lieux hautement symboliques et fortement qualifiés, les vues de la citadelle du Caire et celles qu'elle permet de réaliser sont particulièrement récurrentes dans le corpus iconographique de la ville. Ces documents montrent comment certains espaces urbains peuvent être l'objet de concentrations sémantiques quasi permanentes. A 
l'importance historique du quartier de la citadelle sont venus s'ajouter des projets récents de développement touristique (secteur piétonnier entre les mosquées Sultan Hassan et AlRifa'i, restauration de Bâb al-'Azab, projet de complexe touristique dans l'enceinte basse...). Ces aménagements contribuent eux aussi à l'accroissement de la charge symbolique du quartier. Dans cette escalade de qualification l'iconographie joue un double rôle. Si son existence confirme l'importance symbolique des lieux représentés, sa réalisation peut entraîner de nouvelles qualifications. Les bandes horizontales dont ont été badigeonnées les mosquées du Caire à l'occasion de l'inauguration du canal de Suez en 1869 ont peut-être pour origine celles qui ornent les monuments représentés par les lithographies (en couleur) de David Roberts et de ses successeurs. Enfin, les dessins de Prisse d'Avennes et ceux de P. X. Coste qui montrent des bâtiments épurés, voire restitués, constituent autant de modèles dont les images apparaissent de manière explicite à travers les travaux de restauration menés par le Comité de conservation des monuments de l'Art arabe à la fin du XIXe siècle ${ }^{20}$.

\footnotetext{
${ }^{20}$ La planche 26 du recueil de P. X. Coste, relevée vers 1820, montre devant la qibla de la mosquée Sultan Hassan une rangée de boutiques d'apparence soignée. En 1798, la Description de l'Egypte donne une image plus pitoyable de ces bâtiments. En 1869, ils sont décrits de la manière suivante « des masures sans formes s'adossaient au splendide monument ». Les premières années d'activité du Comité de conservation des monuments de l'Art arabe (1882-1886) sont très occupées à la suppression des boutiques qui masquent la base des monuments. P. X. Coste, Architecture arabe des monuments du Kaire, Paris, 1839. Description de l'Egypte, op. cit., pl. 32. Itinéraire des invités aux fêtes d'inauguration du canal de Suez, Le Caire, 1869. p. 13. Comité de conservation des monuments de l'Art arabe, rapports d'exercice, Le Caire, 1884 à 1887. En 1906, au sujet de la reconstruction de la coupole du tombeau de Al-Ghuri, les membres du Comité se réfèrent explicitement à la représentation de ce tombeau publié par Prisse d'Avenne. Comité de conservation, op. cit., vol. 23, p. 103. Prisse d'Avennes, L'art arabe d'après les monuments du Kaire, Paris, J. Savoy \& Cie, 1877, vol. 1, pl. XXVI.
} 